Tropical Journal of Pharmaceutical Research October 2021; 20 (10): 2143-2148

ISSN: $1596-5996$ (print); 1596-9827 (electronic) (C) Pharmacotherapy Group, Faculty of Pharmacy, University of Benin, Benin City, 300001 Nigeria.

\title{
Effect of a combination of omeprazole and high-dose proton pump inhibitors on the treatment of patients with liver cirrhosis complicated with upper gastrointestinal hemorrhage
}

\author{
Qiang Chi ${ }^{1 *}$, Zhiqiang Lv², Wenjuan Song ${ }^{3}$ \\ ${ }^{1}$ Health Management Center, Qingdao Chengyang District People's Hospital, Chengyang, Qingdao, ${ }^{2}$ Qingdao Chengyang \\ District People's Hospital, Chengyang, Qingdao, ${ }^{3}$ Thoracic \& Cardiovascular Surgery, Qingdao Chengyang District People's \\ Hospital, Chengyang, Qingdao, China \\ *For correspondence: Email: xianweimi01132@163.com; Tel: +86-17806232677
}

\begin{abstract}
Purpose: To determine the effect of a combination of omeprazole and high-dose proton pump inhibitor (PPI) on the treatment of patients with liver cirrhosis complicated with upper gastrointestinal hemorrhage.

Methods: A total of 100 patients with liver cirrhosis and upper gastrointestinal hemorrhage who were admitted to Qingdao Chengyang District People's Hospital from January 2019 to September 2020 were matched and randomly assigned to a control group and a study group. Patients in both groups received a high-dose PPI treatment, while those in the study group were given omeprazole in addition to highdose PPI. Total treatment effectiveness, incidence of adverse reactions, bleeding volume, hemostasis time, liver function after treatment, Quality of Life Index (QLI) scores, Visual Analogue Scale (VAS) scores, and bleeding (\%) at 1, 2 and 4 weeks after treatment were compared for the two groups of patients.

Results: Omeprazole-PPI combination produced a much more favorable outcome than treatment with only high-dose PPI, in terms of effectiveness, QLI scores and liver function $(p<0.05)$. The study group had significantly lower incidence of adverse reactions, bleeding volume, VAS scores, and degree of bleeding at 1, 2 and 4 weeks after treatment, as well as shorter hemostasis time, than the control group $(p<0.05)$.

Conclusion: A combination treatment of omeprazole and high-dose PPI produces better therapeutic effect than high-dose PPI alone, in patients with liver cirrhosis and upper gastrointestinal hemorrhage.
\end{abstract}

Keywords: Omeprazole, High-dose proton pump inhibitor, Liver cirrhosis, Upper gastrointestinal hemorrhage

\begin{abstract}
This is an Open Access article that uses a funding model which does not charge readers or their institutions for access and distributed under the terms of the Creative Commons Attribution License (http://creativecommons.org/licenses/by/4.0) and the Budapest Open Access Initiative (http://www.budapestopenaccessinitiative.org/read), which permit unrestricted use, distribution, and reproduction in any medium, provided the original work is properly credited.

Tropical Journal of Pharmaceutical Research is indexed by Science Citation Index (SciSearch), Scopus, International Pharmaceutical Abstract, Chemical Abstracts, Embase, Index Copernicus, EBSCO, African Index Medicus, JournalSeek, Journal Citation Reports/Science Edition, Directory of Open Access Journals (DOAJ), African Journal Online, Bioline International, Open-J-Gate and Pharmacy Abstracts
\end{abstract}

\section{INTRODUCTION}

Liver cirrhosis is a far commoner liver disease which basically refers to physiological decline in liver functions. In general, liver cancer is invariably complicated with a high possibility of cirrhosis. The liver is an organ that plays a vital role in metabolism. Thus, a decline in liver 
function may trigger complications since the liver is crucial for human life activities and blood circulation [1-3]. One of the complications of liver cirrhosis in the digestive system is upper gastrointestinal hemorrhage due to difficulty in digesting food. This requires timely hemostatic treatment or blood transfusion if necessary, or the patient may suffer shock or even die due to excessive blood loss [4-6]. Proton pump inhibitors (PPIs) are widely used in diseases such as peptic or gastric ulcer, and upper gastrointestinal hemorrhage, and they have advantages of low toxicity and high efficacy. Omeprazole is a PPI which is frequently presented in the form of enteric-coated capsules [7-9]. It has been reported in previous studies that high-dose PPI exerted excellent hemostatic and therapeutic effects against upper gastrointestinal hemorrhage in patients. This study was carried out as an in-depth investigation on the therapeutic effect and clinical application value of combination of omeprazole and high-dose PPI in patients with liver cirrhosis and upper gastrointestinal hemorrhage. These patients were matched as subjects and treated with high-dose PPI or and high-dose PPI combined with omeprazole. Total treatment efficacy was compared with regards to incidence of adverse reactions, bleeding volume, hemostasis time, liver function after treatment, QLI score, VAS score, and degree of bleeding at 1,2 , and 4 weeks after treatment.

\section{METHODS}

\section{General profile of patients}

A total of 100 patients with liver cirrhosis and upper gastrointestinal hemorrhage admitted to
Qingdao Chengyang District People's Hospital from January 2019 to September 2020 were matched and assigned equally to a control group and a study group through random draw, 50 patients each. The ages of patients in the study group ranged from 44 to 71 years, while those in the control group ranged from 45 to 70 years. The general information in the two groups is similar $(p>0.05)$. The baseline information on the patients are listed in Table 1.

\section{Inclusion and exclusion criteria}

\section{Inclusion criteria}

Patients who met the clinical manifestations of liver cirrhosis complicated with upper gastrointestinal bleeding were included: those aged $\geq 18$ years, patients who had no history of drug allergy, drug abuse, or bad habits; patients who had no other organic diseases, and those who signed informed consent forms.

\section{Exclusion criteria}

Patients in the following categories were excluded from the study: those who had a consciousness disorder and could not cooperate in the research, patients who had malignant tumors; and those who did not agree to participate in the study.

The protocol was approved by the Medical Science Research Ethics Committee of Qingdao Chengyang District People's Hospital (approved no. 2017-03(004)) and followed international guidelines for human studies [10].

Table 1: General information on patients in the two groups

\begin{tabular}{lcccc}
\hline Parameter & Study group & Control group & $\boldsymbol{X}^{\mathbf{2}} / \mathbf{t}$ & $\boldsymbol{P}$-value \\
\hline Gender & $23 / 27$ & $26 / 24$ & 0.36 & 0.55 \\
(male/female) & $58.32 \pm 6.21$ & $58.77 \pm 6.39$ & 0.36 & 0.72 \\
Age (years) & $165.32 \pm 10.01$ & $165.64 \pm 10.37$ & 0.16 & 0.88 \\
Height (cm) & $70.03 \pm 5.90$ & $69.38 \pm 5.64$ & 0.56 & 0.57 \\
Weight (kg) & $5.68 \pm 1.69$ & $5.72 \pm 1.73$ & 0.12 & 0.91 \\
$\begin{array}{l}\text { Course of disease } \\
\text { (months) }\end{array}$ & $4.31 \pm 1.33$ & $4.27 \pm 1.38$ & 0.15 & 0.88 \\
$\begin{array}{l}\text { History of Smoking } \\
\text { (years) }\end{array}$ & $10.96 \pm 1.38$ & $10.52 \pm 1.22$ & 1.69 & 0.09 \\
$\begin{array}{l}\text { History of } \\
\text { drinking(years) }\end{array}$ & 13 & 15 & 0.20 & 0.66 \\
$\begin{array}{l}\text { Hypertension } \\
\text { (cases) }\end{array}$ & 8 & 7 & 0.08 & 0.78 \\
$\begin{array}{l}\text { Diabetes (cases) } \\
\text { Hyperlipidemia }\end{array}$ & 4 & 6 & 0.44 & 0.51 \\
(cases) & & & & \\
\hline
\end{tabular}

Values are mean \pm SD 


\section{Treatments}

The control group was treated with high-dose PPI only. On admission, the patients were given $40 \mathrm{mg}$ of pantoprazole (Guangdong Xinghao Pharmaceutical Co. Ltd; NMPA approval no. H20158008; Specification: 40 mg) intravenously, twice a day for three consecutive days. The patients fasted during the medication and received enteral nutrition support. Patients in the experimental group received omeprazole treatment in combination with high-dose PPI. The patients were given $40 \mathrm{mg}$ of omeprazole (Haikou Qili Pharmaceutical Co. Ltd.; NMPA approval no. H20059393; specification: $40 \mathrm{mg}$ ) via intravenous drip, twice times a day for three consecutive days. They fasted during the medication and received enteral nutrition support.

\section{Evaluation of indicators}

Total treatment effectiveness, incidence of adverse reactions, bleeding volume, hemostasis time, liver function after treatment, QLI scores, VAS scores, and extent of bleeding at 1,2 and 4 weeks after treatment were compared between the two groups of patients.

\section{Treatment effectiveness}

Treatment effectiveness was classified as either markedly effective, effective or ineffective. If the upper gastrointestinal hemorrhage was successfully stopped without adverse reactions, the treatment was markedly effective; but if the upper gastrointestinal hemorrhage was successfully stopped, with mild adverse reactions, the treatment was deemed effective. However, if the patients died due to excessive blood loss or the bleeding did not stop for a long time during the treatment, and there was serious adverse reactions, the treatment was ineffective.

\section{Liver function}

The liver function test indicators measured comprised total bilirubin, total protein, total bile acid, alanine aminotransferase (ALT) and aspartate aminotransferase (AST).

\section{VAS score}

The VAS pain scale is based on a total score of 10 points. A score of 0 point indicates no pain, scores of 1 - 3 points indicate tolerable and mild pain, scores of $4-6$ points indicate tolerable but obvious pain, while scores of $7-10$ points reflect severe and intolerable pain [ 10-12].

\section{QLI score}

The QLI includes score standards for daily activities, work and life, and interpersonal relationships. Each standard has a full score of 10 points. The higher the score, the better the patient's quality of life, and vice versa.

\section{Statistical analysis}

All data in this study were analyzed using SPSS 20.0, while GraphPad Prism 7 (GraphPad Software, San Diego, USA) was employed for preparation of graphics. Measurement data are presented as mean \pm standard deviation (SD), and were analyzed using $t$-test. Count data are presented as numbers and percentages [n (\%)], and were analyzed with chi squared $\left(X^{2}\right)$ test. Statistical significance of difference was assumed at $p<0.05$.

\section{RESULTS}

\section{Treatment efficacy}

The results in Figure 1 show a higher treatment effectiveness in the study group than in the control group $(p<0.05)$.

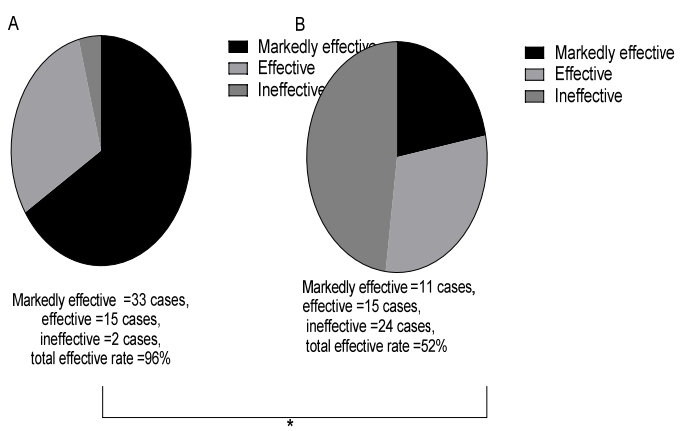

Figure 1: Comparison of treatment efficacy between the two groups Note: $\mathrm{A}=$ study group; while $\mathrm{B}=$ control group

There were 33 markedly effective, 15 effective and 2 ineffective cases in the study group, giving a total effectiveness of $96 \%$ (B). In contrast, total effectiveness in the control group was $52 \%$, comprising 11 markedly effective, and 24 ineffective cases (B). ${ }^{*} P<0.001, \mathrm{X}^{2}=25.16$.

\section{Incidence of adverse reactions}

As shown in Table 2, a strikingly lower incidence of adverse reactions was in the study group than the control group was observed $(p<0.05)$. 
Table 2: Comparison of the incidence of adverse reactions between the two groups

\begin{tabular}{lcccc}
\hline Group & Ascites & Death & Portal hypertension (N) & Total incidence (\%) \\
\hline Study & 1 & 0 & 0 & $2 \%$ \\
Control & 3 & 1 & 8 & $24 \%$ \\
$X^{2}$ & & & 10.70 \\
$P$-value & & & & 0.001 \\
\hline
\end{tabular}

Table 3: Comparison of liver function after treatment between the two groups

\begin{tabular}{llllll}
\hline Group & $\begin{array}{l}\text { Total protein } \\
(\mathbf{g} / \mathbf{L})\end{array}$ & ALT (U/L) & AST (U/L) & $\begin{array}{l}\text { Total } \\
\text { bilirubin } \\
(\boldsymbol{\mu m o l} / \mathbf{L})\end{array}$ & $\begin{array}{l}\text { Total bile acids } \\
(\boldsymbol{\mu m o l} / \mathbf{L})\end{array}$ \\
\hline Study & $73.59 \pm 3.66$ & $49.39 \pm 2.74$ & $56.64 \pm 3.48$ & $18.52 \pm 2.51$ & $7.21 \pm 0.52$ \\
Control & $66.75 \pm 2.51$ & $97.71 \pm 5.68$ & $77.61 \pm 4.45$ & $23.69 \pm 3.30$ & $11.48 \pm 2.23$ \\
$t$ & 10.90 & 54.18 & 26.25 & 7.16 & 13.19 \\
$P$-value & $<0.001$ & $<0.001$ & $<0.001$ & $<0.001$ & $<0.001$ \\
\hline
\end{tabular}

Values are presented as mean \pm SD

\section{Bleeding volume and hemostasis time}

Omeprazole produced a more beneficial effect by reducing bleeding volume and hemostasis time in the study group, when compared with the control group which received PPI only $(p<0.05)$. These results are presented in Figure 2.
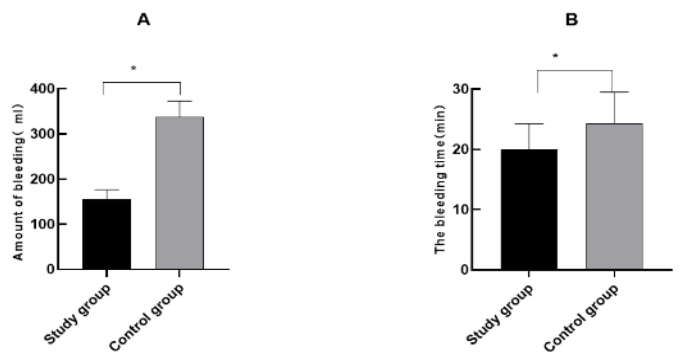

Figure 2: Comparison of bleeding volume and hemostasis time between the two groups. A: bleeding volume in each of the two groups. ${ }^{*} P<0.001$, blood loss in the study group $(156.29 \pm 20.13 \mathrm{~mL})$ compared with that in the control group $(337.58 \pm 35.60 \mathrm{~mL})$. B: hemostasis time in each group. ${ }^{*} P<0.001$, hemostasis time in the study group $(19.98 \pm 4.27 \mathrm{~min})$ compared with that in the control group $(24.40 \pm 5.12$ $\min$ )

\section{Liver function after treatment}

The results in Table 3 reveal that after treatment, the combination of omeprazole and PPI produced better improvement in liver function than PPI alone $(p<0.05)$.

\section{QLI and VAS scores}

As shown in Figure 3, the study group was witnessed with a significantly lower VAS score, and markedly higher QLI score in contrast to the control group $(p<0.05)$.

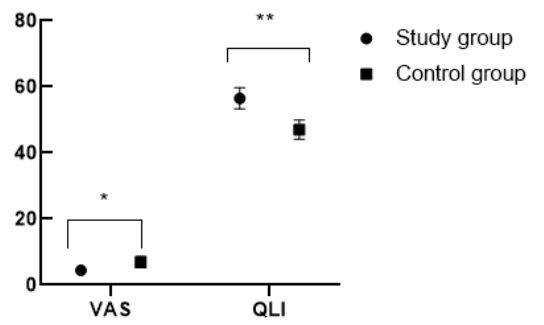

Figure 3: Comparison of post-treatment scores in QLI and VAS between the two groups. ${ }^{*} P<0.001$, VAS score in the study group $(4.41 \pm 1.33)$ compared with that in the control group (6.92 \pm 1.69$) .{ }^{* \star} P<0.001$, QLI score in the study group $(56.51 \pm 3.22)$ compared with that in the control group $(47.04 \pm 2.85)$

\section{Bleeding at 1 week, 2 weeks, and 4 weeks post-treatment}

Follow-up records of patients after treatment, as displayed in Figure 4, revealed a more favorable outcome on \% bleeding at 1,2 and 4 weeks after treatment in the study group of patients (omeprazole group) than in the control group that received $\mathrm{PPI}$ treatment only $(p<0.05)$.

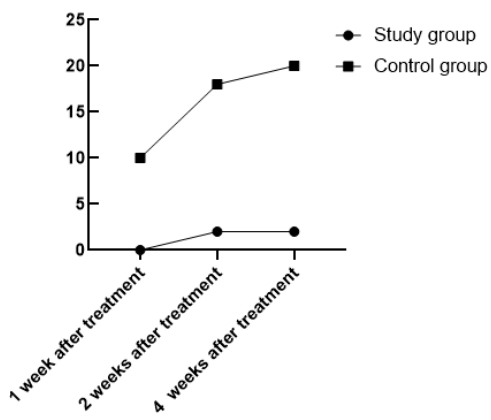

Figure 4: Comparison of bleeding post-treatment between the two groups. Ordinate $=\%$ bleeding 
There was significantly lower \% bleeding at each time point in the study group than in the control group, i.e., after 1, 2 and 4 weeks ( $p=0.02, p=$ $0.008, p=0.004$, respectively).

\section{DISCUSSION}

Upper gastrointestinal hemorrhage is one of the common complications in the treatment of liver cirrhosis patients. The weakened liver function in patients with liver cirrhosis invariably leads to symptoms such as portal hypertension and vasodilation. As a result, difficulty in digesting food, or difficulty in swallowing food may easily produce hemorrhage in the digestive tract. [13]. Patients with mild gastrointestinal bleeding need only to take certain hemostatic measures. However, such measures are ineffective if the bleeding is severe. Thus, in such a case, timely blood transfusion combined with drug treatment is indispensable [14]. It is generally accepted that PPI, one of the frequently used drugs in the treatment of diseases such as gastrointestinal ulcers and gastrointestinal bleeding, yields a favorable outcome in clinical trials on gastrointestinal hemorrhage, but with slight side effects [15].

The current study was being undertaken with an attempt to conduct an in-depth investigation on the clinical effect of combination of omeprazole and high-dose PPI in patients with liver cirrhosis and upper gastrointestinal bleeding, and to analyze the impact of the treatment on quality of life and other indicators. The results demonstrate that combined treatment with omeprazole and high-dose PPI produced a much more favorable outcome than only high-dose PPI treatment in terms of treatment effectiveness, QLI scores and liver function after treatment. The liver is an important organ involved in detoxification and metabolism and regulation of various functions in the human body. Some very important indicators of liver function are ALT, AST, total protein, total bilirubin, and total bile acids. If tests of liver function result in levels of these indicators in excess of the normal ranges, impairment of liver function and higher risks of liver diseases are implied [16,17].

In this study, the combination of omeprazole and high-dose PPI treatment produced little or no changes in the liver function of enrolled patients, indicating the safety of this treatment. The experimental group had lower incidence of adverse reactions, bleeding volume, VAS scores, and percentage bleeding at 1, 2, and 4 weeks after treatment, as well as shorter hemostasis time, than the control group. Severe upper gastrointestinal bleeding may result in shock or even death. Thus, it requires timely blood transfusion to ensure sufficient blood volume for normal body functions. Accordingly, monitoring and recording of bleeding volume not only determine whether the blood transfusion is needed so as to understand the degree of patient's gastrointestinal bleeding, it also serves as one key method for judging whether hemostatic measures are reasonable and effective. Since liver cirrhosis complicated by upper gastrointestinal hemorrhage is triggered by lesions in the relatively fragile digestive tract, the reoccurrence of bleeding after treatment is rather frequent. Therefore, follow-up of bleeding within 4 weeks after treatment is vital not only for improving and optimizing treatments, but also for identifying how to arrest such bleeding in the future.

The combination of omeprazole and high-dose PPI resulted in a more desirable outcome in bringing down the bleeding volume, hemostasis time and furthermore, the risk of reoccurrence of bleeding after treatment. The findings of Liu et al [18] further support the feasibility of this study, which showed better hospitalization time, bleeding at $48 \mathrm{~h}$ after treatment, hemostasis time, and blood transfusion volume in the experimental group, indicating that pantoprazole produced a superior treatment efficacy.

\section{Limitations of the study}

Some limitations of the current trial should be highlighted, for instance, it is probably argued by the enrolled participants whom are from similar background. Thus, larger number of trial samples is expected to be recruited from different regions.

\section{CONCLUSION}

The combination of omeprazole with high-dose PPI produces enhanced therapeutic effects in patients with liver cirrhosis and upper gastrointestinal hemorrhage. Thus, the combined treatment has clinical merit.

\section{DECLARATIONS}

\section{Conflict of Interest}

No conflict of interest associated with this work.

\section{Contribution of Authors}

The authors declare that this work was done by the authors named in this article and all liabilities pertaining to claims relating to the content of this article will be borne by them.

Trop J Pharm Res, October 2021; 20(10): 2147 


\section{Open Access}

This is an Open Access article that uses a funding model which does not charge readers or their institutions for access and distributed under the terms of the Creative Commons Attribution License (http://creativecommons.org/licenses/by/ 4.0) and the Budapest Open Access Initiative (http://www.budapestopenaccessinitiative.org/rea d), which permit unrestricted use, distribution, and reproduction in any medium, provided the original work is properly credited.

\section{REFERENCES}

1. Price E, Treacher DF. Reduced gastric acidity, proton pump inhibitors and increased severity of COVID-19 infections. Crit Care. 2021; 25 (1): 73.

2. Wu B, Li D, Xu T, Luo M, He Z, Li Y. Proton pump inhibitors associated acute kidney injury and chronic kidney disease: data mining of US FDA adverse event reporting system. Sci Rep. 2021; 11 (1): 3690.

3. Labenz C, Schattenberg JM. Letter: proton pump inhibitor use and bone fracture risk-a mechanistic point of view. Authors' reply. Aliment Pharmacol Ther. 2021; 53 (5): 673.

4. Rocco A, Compare D, Sgamato C, Coccoli P, Nardone G. Editorial: proton pump inhibitor use in cirrhosis-a piece of the puzzle. Authors' reply. Aliment Pharmacol Ther. 2021; 53: 665-666.

5. Celsa C, Cammà C. Editorial: proton pump inhibitor use in cirrhosis-a piece of the puzzle. Aliment Pharmacol Ther. 2021; 53: 663-664.

6. Sugiyama T. Letter: proton pump inhibitor use and bone fracture risk-a mechanistic point of view. Aliment Pharmacol Ther. 2021; 53(5): 671-672.

7. Lai SW. Proton pump inhibitors and the risk of pancreatic cancer. J Gastroenterol. 2021; 56 (3): 293-294.

8. He W, Shu $X$, Zhu E, Deng B, Lin $Y$, Wu X, Zhou Z, Wang $J$, Nie $R$. Intermittent concurrent use of clopidogrel and proton pump inhibitors did not increase risk of adverse clinical outcomes in Chinese patients with coronary artery disease. BMC Cardiovasc Disord. 2021; 21 (1): 75.

9. Garcia-Mazcorro JF, Suchodolski JS, Jones KR, ClarkPrice SC, Dowd SE, Minamoto Y, Markel M, Steiner JM, Dossin O. Effect of the proton pump inhibitor omeprazole on the gastrointestinal bacterial microbiota of healthy dogs. FEMS Microbiol Ecol. 2012; 80 (3): 624-36.

10. Department of Health, Education, and Welfare; National Commission for the Protection of Human Subjects of Biomedical and Behavioral Research. The Belmont Report. Ethical principles and guidelines for the protection of human subjects of research. J Am Coll Dent 2014; 81: 4-13.

11. Brusselaers N, Sadr-Azodi O, Engstrand L. Proton pump inhibitors and the risk of pancreatic cancer. J Gastroenterol. 2021; 56 (3): 295-296.

12. Neumann I, Letelier LM, Rada G, Claro JC, Martin J, Howden CW, Yuan $Y$, Leontiadis GI. Comparison of different regimens of proton pump inhibitors for acute peptic ulcer bleeding. Cochrane Database Syst Rev. 2013;12 (6): CD007999.

13. Yoshikawa M, Kinoshita $H$, Nishimura N, Takai $R$, Matsuda T, Nakatani S, Shioyama E, Takeda K, Yoshiji H. A surgically treated case of severe upper gastrointestinal hemorrhage with gastritis cystica polyposa. BMC Gastroenterol. 2021; 21 (1): 27.

14. Ahmed G, Abid M, Hosmane S, Mathew S. Unusual case of upper gastrointestinal haemorrhage secondary to a ruptured gastroduodenal artery pseudoaneurysm: case presentation and literature review. BMJ Case Rep. 2020; 13 (11): e236463.

15. Shah N, Chen C, Montano N, Cave D, Siegel R, Gentile NT, Limkakeng AT Jr, Kumar AB, Ma Y, Meltzer AC. Video capsule endoscopy for upper gastrointestinal hemorrhage in the emergency department: A systematic review and meta-analysis. Am J Emerg Med. 2020; 38 (6): 1245-1252.

16. Driver BE, Horton G, Barkun A, Martel M, Klein LR. Validation of a decision rule to predict patients at low risk of variceal upper gastrointestinal hemorrhage. Am J Emerg Med. 2021; 44: 267-271.

17. Edhi Al, Hakim S, Shams C, Bedi D, Amin M, Cappell MS. Clarithromycin-Associated Acute Liver Failure Leading to Fatal, Massive Upper Gastrointestinal Hemorrhage from Profound Coagulopathy: Case Report and Systematic Literature Review. Case Reports Hepatol. 2020; 2020:2135239.

18. Liu J, Wang G, Liu Y, Huang L, Xu X, Wang J. Effects of Somatostatin Combined with Pantoprazole on Serum CReactive Protein and Intercellular Adhesion Molecule-1 in Severe Acute Pancreatitis. J Coll Physicians Surg Pak. 2019; 29 (7): 683-684. 